\title{
Dynamics of Primary Charge Separation in Bacterial Photosynthesis Using the Multilevel Redfield-Davies Secular Approach
}

\author{
ROVSHAN G. SADYGOV, DANIEL NEUHAUSER \\ Department of Chemistry and Biochemistry, University of California, Los Angeles, \\ California 90095-1569
}

Received 15 May 2001; revised 6 July 2001; accepted 20 August 2001

\begin{abstract}
We use Redfield's relaxation theory in the Davies formulation to study primary charge separation reactions in bacterial photosynthesis. The specific model studied is the standard one (spin-boson), with three states for the system, harmonic oscillators for the bath, and linear ohmic system-bath coupling. The Redfield-Davies formulation, which is equivalent to the secular approximation, is Markovian, of second order in system-bath coupling, and is written for the system's density matrix. The approximation does not suffer from any negative probabilities (which appear in the original Redfield approach) and can therefore be used for long-time processes ( $20 \mathrm{ps}$ or more here). Our results are in line with previous studies, especially to high bath frequencies. They confirm the usefulness of the Redfield-Davies secular approach as a convenient and simple tool for studying system-bath processes. () 2002 Wiley Periodicals, Inc. Int J Quantum Chem 87: 254-263, 2002
\end{abstract}

Key words: electron transfer; density matrix; system-bath coupling; Redfield theory; Davies' device

\section{Introduction}

o ne of the most intensively studied electron transfer (ET) reactions in biological systems is the primary charge separation in bacterial reedu.

Correspondence to: R. G. Sadygov; e-mail: rovshan@scripps.

Contract grant sponsors: NSF, PRF, Sloan Foundation, and UCLA Academic Senate. action centers (RCs) (Rhodopseudomonas viridis) [1]. Much of the focus of the research has been on ET from an excited primary donor, $P^{*}$-a pair of strongly coupled bacteriochlorophylls-to bacteriopheophytin, $H_{\mathrm{L}}$, which takes about 3.5 ps (Rhodobacter sphaeroides) $[2,3]$. The center-to-center distance between these chromophores is $17 \AA$. Due to the exponential scaling of the coupling with the distance, direct ET over such a distance with such a fast rate seems implausible. The transfer is facilitated instead by the presence of a monomer of the 
bacteriochlorophyll molecule, $B_{\mathrm{L}}$, located between the chromophores. The center-to-center distances from $B_{\mathrm{L}}$ to $P$ and $H_{\mathrm{L}}$ are $\sim 13$ and $11 \AA$, respectively. The primary charge separation process in bacterial photosynthesis is therefore a multilevel process involving at least three states: the donor state, $P^{*} B_{\mathrm{L}} H_{\mathrm{L}}$; the intermediate or bridge state, $P^{+} B_{\mathrm{L}}^{-} H_{\mathrm{L}}$; and the acceptor state, $P^{+} B_{\mathrm{L}} H_{\mathrm{L}}^{-}$.

Two alternative mechanisms ("sequential" and "superexchange") have been suggested for the role of $B_{\mathrm{L}}$ in the primary charge separation: a two-step sequential process

$$
P^{*} B_{\mathrm{L}} H_{\mathrm{L}} \rightarrow P^{+} B_{\mathrm{L}}^{-} H_{\mathrm{L}} \rightarrow P^{+} B_{\mathrm{L}} H_{\mathrm{L}}^{-}
$$

in which the electron resides a finite time on the monomer bacteriochlorophyll molecule, and a single-step, superexchange mechanism

$$
P^{*} B_{\mathrm{L}} H_{\mathrm{L}} \rightarrow P^{+} B_{\mathrm{L}} H_{\mathrm{L}}^{-},
$$

where virtual orbitals of $B_{\mathrm{L}}$ are used to transport the electron.

There have been a number of theoretical studies of this problem [4-9]. Electronic structure calculations [4] determined that the two lowest excited states of $P$ are exciton states split by approximately $1300 \mathrm{~cm}^{-1}$. Molecular dynamics simulations using these electronic structure results produced bath response functions in the linear response limit [5]. The calculations supported the superexchange mechanism.

Egger and Mak [6, 7] employed a Monte Carlo path integrals method to study primary charge separation in RCs within the three-state spin-boson model (i.e., within a model which assumes that the electronic degrees of freedom can be represented by the three states, while the thermal fluctuations are described by a collection of harmonic oscilators coupled to the electronic motion). Ohmic spectral density was used for the bath, and the simulations were carried out up to 2 ps. The authors suggested a set of intrasystem electronic and system-protein coupling parameters which would reproduce the experimentally observed reaction rates.

In an alternative approach Makri and co-workers have shown that for systems near the Markovian limit, the path integrals for the spin-boson model are reduced to a series of matrix multiplications $[10,11]$. They applied the method to the ET in photosynthetic RCs $[8,9]$. A set of parameters have been obtained, which facilitates a fast reaction rate. It was concluded that the sequential mechanism greatly promotes ET. Later electronic structure cal- culations [12] of the RC chromophores produced values in support of these dynamics calculations.

Both these approaches are in principle exact but numerically intensive. It is therefore desirable to examine approximate methods which scale gently with problem size and therefore are able to handle larger systems. One such approach was recently suggested by Friesner et al. [13], based on the linearization of Makri's matrix-transfer method. Other approaches include semiclassical simulations [14] and the secular approximation, equivalent to Davies' formulation of the Redfield theory. Here we examine the latter and find that it gives qualitatively correct results in the high bath frequency limit, in good agreement with the rigorous path integral calculations. The temperature dependence of the reaction rate from our calculations is the same as those from the work of Makri and coworkers [8, 9]. Long-time dynamics are also similar to the path-integral simulations. However, some differences emerge for short-time dynamics, and the method fails for low-frequency baths.

The most widely used method for approximate description of bath-system dynamics is the Redfield theory [15-20]. This theory describes the systembath dynamics directly in terms of the system's density matrix, using second-order perturbation theory for propagating the system's density matrix with time. Originally the Redfield approach was developed for nuclear magnetic resonance $[15,16]$. Recently it has been used in optical spectroscopy as well. It was previously adopted to study aspects of ET processes in biological systems such as DNA $[17,21]$ and RCs of photosynthetic bacteria [22]. In these studies the quantum bath correlation functions were replaced by classical correlation functions. As is the normal practice, a thermal prefactor was added to ensure that the detailed balance condition was satisfied. The work predicted the possibility of a long-range electron transfer in DNAs. However, as was pointed out [18] the approach does not reduce to Marcus theory in the proper limit. Even more severely, the positive definiteness of the density matrix is not conserved in the original Redfield theory. This creates a problem in treating the diagonal elements of the density matrix as population numbers of the corresponding states.

In this article we report results of our study on the primary charge separation in bacterial photosynthesis using the secular approximation, also known as the Davies' formulation of the Redfield theory. Davies suggested a device which preserves the positive-definite character of the system density 
matrix [23, 24]. It allows the use of quantum bath correlation functions which account for the medium reorganization energy and enables us to present long-time dynamics of the reaction rate and its temperature dependence.

The plan of the article is as follows. The next section briefly describes the model Hamiltonian, the Redfield formalism, and Davies' device. Section 3 presents results and a discussion. Section 4 summarizes and concludes.

\section{Methodology}

\subsection{MODEL}

The total Hamiltonian is a sum of system and bath Hamiltonians and a system-bath interaction potential

$$
H_{\mathrm{T}}=H_{\mathrm{S}}+H_{\mathrm{b}}+V_{\mathrm{Sb}} .
$$

The Hamiltonian chosen is a three-state, of spinboson type [25], extensively used in studies of the primary charge separation processes in bacterial photosynthesis $[6,9]$. The three-state system part is

$$
H_{\mathrm{S}}=\left(\begin{array}{lll}
H_{11} & H_{12} & H_{13} \\
H_{21} & H_{22} & H_{23} \\
H_{31} & H_{32} & H_{33}
\end{array}\right) .
$$

The diagonal elements of the system Hamiltonian correspond to the primary donor, $P^{*} B_{\mathrm{L}} H_{\mathrm{L}}$, bridge, $P^{+} B_{\mathrm{L}}^{-} H_{\mathrm{L}}$, and acceptor states, $P^{+} B_{\mathrm{L}} H_{\mathrm{L}}^{-}$. In the calculations below $H_{11}$ is set equal to zero. Nondiagonal elements of $H_{\mathrm{S}}$ arise due to electronic couplings: $H_{12}$ is the coupling between $P^{*} B_{\mathrm{L}} H_{\mathrm{L}}$ and $\mathrm{P}^{+} B_{\mathrm{L}}^{-} H_{\mathrm{L}}$ and $H_{23}$ is the coupling between $P^{*} B_{\mathrm{L}} H_{\mathrm{L}}$ and $P^{+} B_{\mathrm{L}}^{-} H_{\mathrm{L}}$. Due to the large intermolecular distance the electronic coupling of $P^{*} B_{\mathrm{L}} H_{\mathrm{L}}$ and $P^{+} B_{\mathrm{L}} H_{\mathrm{L}}^{-}, H_{13}$, has been set equal to zero.

The bath degrees of freedom are modeled as harmonic oscillators:

$$
H_{\mathrm{b}}=\sum_{\alpha} \frac{p_{\alpha}^{2}}{2 m_{\alpha}}+\frac{m_{\alpha} \omega_{\alpha}^{2} x_{\alpha}^{2}}{2} .
$$

The system-bath coupling is assumed to be a product of system, $S$, and bath, $B$, functions and is linear in bath displacements from their equilibrium:

$$
V_{\mathrm{Sb}}=S \cdot B=\left(\begin{array}{ccc}
-1 & 0 & 0 \\
0 & 0 & 0 \\
0 & 0 & 1
\end{array}\right) \cdot \sum_{\alpha} C_{\alpha} x_{\alpha}
$$

where $C_{\alpha}$ is the coupling constant of the system to the $\alpha$ th mode of the bath. In forthcoming studies we plan to consider additional effects such as inelastic ET (nonzero nondiagonal elements in the systembath coupling matrix), multidimensional potential energy surfaces and vibrational effects, and Hamiltonians beyond the Condon approximation (i.e., distance-dependent nondiagonal elements of the system Hamiltonian).

The original system Hamiltonian, Eq. (4), is nonadiabatic (nonzero nondiagonal elements). In the next section we will mostly work in the interaction representation, which is more easily presented with the adiabatic system Hamiltonian, $H_{S}^{\alpha}$ :

$$
H_{\mathrm{S}}^{\alpha}=A^{-1} H_{\mathrm{S}} A
$$

where $A$ is a unitary matrix consisting of eigenfunctions of $H_{\mathrm{S}}$. The system-bath coupling, Eq. (6), is transformed similarly and becomes nondiagonal. For simplicity, we drop the superscripts and work in an adiabatic basis unless otherwise indicated. Note here that the nondiagonal elements of the transformation matrix, $A$, are very small due to the large energy separation and weak couplings in the system. Therefore, the density matrices in the adiabatic and diabatic representations do not differ significantly.

In the case of linear system-bath coupling the bath dynamics is completely characterized by the bath spectral density, $J(\omega)$ [26],

$$
J(\omega)=\frac{\pi}{2} \sum_{\alpha} \delta\left(\omega-\omega_{\alpha}\right) \frac{C_{\alpha}^{2}}{\omega_{\alpha}} .
$$

In the simulation of the bacterial RCs an ohmic spectral density is often used:

$$
J(\omega)=\frac{\eta \hbar \omega \pi}{2} \exp \left(-\omega / \omega_{\mathrm{c}}\right),
$$

where $\eta$ is a Kondo factor and $\omega_{\mathrm{c}}$ is a cut-off frequency. The value of $\eta$ characterizes the systembath coupling strength, while $\omega_{\mathrm{c}}$ is the overall system response frequency. The response function [26] is defined by the spectral density via the relationship

$$
\begin{aligned}
\alpha(t)=\frac{1}{\pi} \int_{0}^{\infty} d \omega J(\omega)\left(\operatorname{coth}\left(\frac{1}{2} \hbar \omega \beta\right)\right. & \cos (\omega t) \\
& -i \sin (\omega t)) .
\end{aligned}
$$

In the calculations below we use an ohmic spectral density with parameters that were obtained from molecular dynamics simulations on RCs [5].

The values of $\eta(=1.67)$ and $\omega_{\mathrm{c}}\left(\hbar \omega_{\mathrm{c}}=600 \mathrm{~cm}^{-1}\right)$ determined from the molecular simulation calculations [5] produce a reorganization energy, $\lambda_{r}$, of 
$2000 \mathrm{~cm}^{-1}$ through the relationship

$$
\lambda_{\mathrm{r}}=\frac{4}{\pi} \int_{0}^{\infty} \frac{J(\omega)}{\omega} d \omega .
$$

The above formula for the reorganization energy follows from the definition of the spectral density and the form of the system-bath coupling operator. The $2000 \mathrm{~cm}^{-1}$ energy is the energy gap between donor and acceptor states in RCs of wild-type bacteria [27]. By choosing the reorganization energy equal to the energy difference we have assumed an activationless electron transfer from $P^{*} B_{\mathrm{L}} H_{\mathrm{L}}$ to $P^{+} B_{\mathrm{L}} H_{\mathrm{L}}^{-}$.

\subsection{REDFIELD THEORY}

The Redfield formalism $[15,16]$ as it applies to ET reactions has been discussed extensively in a recent review [28]. Detailed analysis of the factorization of the Redfield tensor into matrices dependent only on bath and system variables are presented there. Here we mostly follow van Kampen [29] for a brief review of Redfield's theory and Davies' device. For a detailed description of the secular approximation, i.e., Davies' approach to Markovian processes and its mathematical foundations, refer to Refs. [23, 24].

The Redfield theory is of the second order of perturbation in system-bath coupling. The first step is the quantum Liouville equation of motion for the total density matrix of the system plus bath, $\rho_{\mathrm{T}}(t)$ :

$$
\dot{\rho}_{\mathrm{T}}(t)=-\frac{i}{\hbar}\left[H_{\mathrm{T}}, \rho_{\mathrm{T}}\right] \equiv \mathcal{L}_{\mathrm{T}} \rho_{\mathrm{T}},
$$

where $\mathcal{L}_{\mathrm{T}}$ is a superoperator in Liouville space. The exact time evolution of the density matrix is given in two equivalent forms:

$$
\begin{aligned}
\rho_{\mathrm{T}}(t)=\exp \left(t \mathcal{L}_{\mathrm{T}}\right) \rho_{\mathrm{T}}(0) \equiv \exp ( & \left.-\frac{i}{\hbar} t H_{\mathrm{T}}\right) \rho_{\mathrm{T}}(0) \\
& \times \exp \left(\frac{i}{\hbar} t H_{\mathrm{T}}\right) .
\end{aligned}
$$

The next step is to solve Eq. (12) perturbatively, and in the second-order part of the perturbation to insert the simplifying assumption that the density matrix of the full system plus bath, $\rho_{\mathrm{T}}(t)$, factors out at all times into system, $\sigma(t)$, and bath, $\rho_{\mathrm{b}}(t)$, density matrices:

$$
\rho_{\mathrm{T}}(t)=\sigma(t) \rho_{\mathrm{b}}(0) \equiv \sigma(t) \rho_{\mathrm{b}}^{\mathrm{e}} .
$$

The bath density matrix is assumed to be time independent and in thermal equilibrium, $\rho_{\mathrm{b}}^{\mathrm{e}}$ (basic condition of irreversibility). This assumption is motivated by the fact that the bath is infinitely large compared to the system and is not affected by it. The condition implies that the system does not "remember" its past (Markovian process) or that bath modes equilibrate much faster than system modes.

With this Markovian assumption, the Redfield theory reduces the quantum Liouville equation for the system plus bath density matrix into the quantum master equation for the system density matrix $[29,30]$ :

$$
\dot{\sigma}(t)=\left(\mathcal{L}_{\mathrm{S}}+\mathcal{K}\right) \sigma(t),
$$

where $\mathcal{L}_{\mathrm{S}}$ is a system superoperator and the operator $\mathcal{K}$ is

$$
\mathcal{K}=\int_{0}^{\infty} d \tau \operatorname{Tr}_{\mathrm{b}}\left(\mathcal{L}_{\mathrm{I}} \mathcal{L}_{\mathrm{I}}(-\tau) \rho_{\mathrm{b}}^{\mathrm{e}}\right),
$$

where $\mathcal{L}_{\mathrm{I}}$ is a system-bath coupling superoperator and $\mathcal{L}_{\mathrm{I}}(-\tau)$ is its interaction representation:

$$
\mathcal{L}_{\mathrm{I}}(-\tau)=\exp \left(\tau\left(\mathcal{L}_{\mathrm{S}}+\mathcal{L}_{\mathrm{b}}\right)\right) \mathcal{L}_{\mathrm{I}} \exp \left(-\tau\left(\mathcal{L}_{\mathrm{S}}+\mathcal{L}_{\mathrm{b}}\right)\right),
$$

which can be rewritten after several steps as

$$
\dot{\sigma}(t)=-\frac{i}{\hbar}\left[H_{S}, \sigma(t)\right]-\{[S, \tilde{S} \sigma(t)]+\text { h.c. }\},
$$

where $\tilde{S}$ is determined from

$$
\tilde{S}_{i j}=\frac{S_{i j}}{\hbar^{2}} \int_{0}^{\infty} d \tau \exp \left(-i \omega_{i j} \tau\right) \alpha(\tau) .
$$

Eq. (18) is derived by using the properties of the superoperators Eqs. (12) and (13) and the systembath coupling Eq. (6). By using those equations one writes out $[28,31]$ the Redfield equation in superoperator form:

$$
\dot{\sigma}_{i j}(t)=-i \omega_{i j} \sigma_{i j}(t)+\sum_{k l} \mathcal{K}_{i j k l} \sigma_{k l}(t),
$$

where the relaxation matrix elements, $\mathcal{K}_{i j k l}$, are defined as

$$
\begin{aligned}
\mathcal{K}_{i j k l}=(i j|\mathcal{K}| k l)= & \tilde{S}_{i k} S_{l j}+\tilde{S}_{j l}^{*} S_{i k} \\
& -\delta_{l j} \sum_{r} S_{i r} \tilde{S}_{r k}-\delta_{i k} \sum_{r} \tilde{S}_{r l}^{*} S_{r j} .
\end{aligned}
$$

$\mathcal{K}_{i j k l}$ are in general complex valued. As is seen from Eq. (20), the imaginary parts of $\mathcal{K}_{i j k l}$ are frequency shifts in the system due to the coupling to the bath, while the real parts are the relaxation constants. The secular equation associated with the Redfield equation is singular of rank 3 [32]: in practice this means that the trace of the density matrix is a conserved quantity but the density matrix is not positive definite. In the next section we will use the Redfield equation in form of Eq. (20) to obtain Davies' device. 


\subsection{DAVIES' DEVICE}

As noted before $[28,29]$ and as we show below, Redfield's theory may lead to negative values for the diagonal elements of the density matrix. This property complicates treating the diagonal elements as population numbers. It has been argued [33] that the only Markovian approximation consistent with the positivity of the dynamics is Davies' theory $[23,34]$, also sometimes called Davies' device or the secular approximation. In this approach the superoperator $\mathcal{K}$ is replaced by its time average; i.e.,

$$
\overline{\mathcal{K}}=\lim _{T \rightarrow \infty} \frac{1}{2 T} \int_{-T}^{T} d t_{1} \exp \left(-t_{1} \mathcal{L}_{\mathrm{S}}\right) \mathcal{K} \exp \left(t_{1} \mathcal{L}_{\mathrm{S}}\right) .
$$

The quantum master equation in Davies' theory becomes

$$
\dot{\sigma}(t)=\left(\mathcal{L}_{\mathrm{S}}+\overline{\mathcal{K}}\right) \sigma(t)
$$

The time averaging of the superoperator $\mathcal{K}$ eliminates oscillating terms $\sim \exp \left(i\left(\omega_{i j}+\omega_{l k}\right) t\right)$ in the Redfield equation:

$$
\begin{aligned}
(i j|\overline{\mathcal{K}}| k l) & =\lim _{T \rightarrow \infty} \frac{1}{2 T} \int_{-T}^{T} d t_{1}\left(i j \mid \exp \left(-t_{1} \mathcal{L}_{\mathrm{S}}\right) \mathcal{K}\right. \\
& \equiv \delta\left(\omega_{i j}+\omega_{l k}\right) \mathcal{K}_{i j k l,}
\end{aligned}
$$

where the last $\delta$ function should be interpreted as a Kronecker delta. In application to our Hamiltonian, Eq. (3), the Davies' device produces the following equation of motion for reduced density matrix:

$$
\begin{aligned}
\dot{\sigma}_{i j}(t)= & -\frac{i}{\hbar}\left[H_{\mathrm{S}}+H^{\prime}, \sigma(t)\right]_{i j} \\
& +\sum_{k l} \delta\left(\omega_{i j}+\omega_{l k}\right)\left(\tilde{S}_{i k} S_{l j}+S_{i k} \tilde{S}_{j l}^{*}\right) \sigma_{k l}(t) \\
& -\sum_{k}\left(S_{i k} \operatorname{Re} \tilde{S}_{k i}+S_{k j} \operatorname{Re} \tilde{S}_{k j}\right) \sigma_{i j}(t),
\end{aligned}
$$

where

$$
H_{i j}^{\prime}=\hbar \sum_{r} S_{i r} \operatorname{Im} \tilde{S}_{r i}
$$

Eq. (25) has a simple interpretation: the coupling terms under the $\sum_{k l}$ and $\sum_{k}$ are real because of the delta symbol and the definition of $\tilde{S}$, Eq. (19). Thus, the system relaxation is due to this term. All frequency shifts in the system due to the coupling to the bath are included in the $H^{\prime}$ (imaginary) term.

Lindblad [35] derived a general form of the equation of motion for the density matrix which preserves its positivity. As is well known, Davies' device is a specific example of this form [29]. To show this we recast Eq. (25) in the following form:

$$
\begin{aligned}
\dot{\sigma}(t)=-\frac{i}{\hbar}\left[H_{S}\right. & \left.+H^{\prime}, \sigma(t)\right]+\frac{1}{\hbar^{2}} \sum_{\omega} \operatorname{Re}(\theta(\omega)) \\
& \times\left\{\left[S_{\omega} \sigma(t), S_{\omega}^{*}\right]+\left[S_{\omega}, \sigma(t) S_{\omega}^{*}\right]\right\},
\end{aligned}
$$

where

$$
S_{\omega}=\sum_{\omega_{k l}=\omega} S_{k l}|k\rangle\langle l|
$$

and

$$
\theta\left(\omega_{k l}\right)=\int_{0}^{\infty} d \tau \exp \left(-i \omega_{k l} \tau\right) \alpha(\tau)
$$

Eq. (27) is of the form first established by Lindblad. This type of equation conserves the positive-definite character of the density matrix $[35,36]$. The Lindblad equation may also be obtained by constructing a general Schrödinger-Langevin equation for wave function and estimating a density matrix at two close times $t$ and $t+\delta t$ [29].

Davies' device has a very simple form in a nonharmonic system, as considered here. The frequency spacings in this system are generally not equal (i.e., $\omega_{21} \neq \omega_{32}$, etc.). Thus, the delta function symbol in Eq. (24) has a zero argument only in two cases. For nondiagonal elements $(i \neq j)$, the argument would vanish only if $k=i$ and $l=j$, while for diagonal elements $(i=j)$, it is necessary that $l=k$. In short, the only elements that are retained are of the form $\mathcal{K}_{\text {iikk }}$ and $\mathcal{K}_{i j i j}$. This is illustrated in the following equations of motion for $\sigma_{i i}(t)$,

$$
\begin{aligned}
& \dot{\sigma}_{i i}(t)= \sum_{k} \mathcal{K}_{i i k k} \sigma_{k k}(t) \\
& \equiv \sum_{k \neq i}\left(\left(\tilde{S}_{i k} S_{k i}+S_{i k} \tilde{S}_{i k}^{*}\right) \sigma_{k k}(t)\right. \\
&\left.\quad-\left(S_{i k} \operatorname{Re} \tilde{S}_{k i}+S_{k i} \operatorname{Re} \tilde{S}_{k i}\right) \sigma_{i i}(t)\right),
\end{aligned}
$$

and for $\sigma_{i j}(t)$,

$$
\begin{aligned}
\dot{\sigma}_{i j}(t)= & -i \omega_{i j} \sigma_{i j}+\mathcal{K}_{i j j i} \sigma_{i j}(t) \\
\equiv & \left(-i\left(\omega_{i j}+H_{i i}^{\prime}-H_{j j}^{\prime}\right)-\sum_{k \neq i} S_{i k} \operatorname{Re} \tilde{S}_{k i}\right. \\
& \left.\quad-\sum_{k \neq j} S_{k j} \operatorname{Re} \tilde{S}_{k j}\right) \sigma_{i j} .
\end{aligned}
$$

Davies' device essentially amounts to the "secular" approximation [31]; i.e., the equations of motion for the diagonal and nondiagonal density matrix elements are uncoupled. Therefore, the coefficients in the equations of motion for diagonal elements can be considered as reaction rates. Note however that 
in our application these equations refer to the adiabatic representation.

Below we will mostly report results obtained by using Davies' device. For a comparison a figure characteristic of Redfield theory results will be presented. For both of the approaches Chebyshev polynomials $[37,38]$ have been used to propagate the density matrix. Numerical issues related to propagation of the Liouville equation have been recently discussed in the literature $[39,40]$.

\section{Results}

At present not all of the parameters characterizing ET in bacterial photosynthesis are known. However, thanks to a large number of studies of this process a set of parameters most efficient in promoting ET has been emerging. We test our approach by using these parameters. Besides confirming the previous conclusions our study also reveals some new features. As was discussed above, we take the reorganization energy equal to the energy difference between donor and acceptor states, $\Delta \mathcal{G}_{31}=H_{33}-$ $H_{11}=-2000 \mathrm{~cm}^{-1}$ [41], corresponding to activationless ET. Given the reorganization energy, the ohmic spectral density is characterized by one parameter only. It may be either the cut-off frequency at which the spectral density is maximum or the system-bath coupling coefficient. In dynamics simulations two sets had been used for these parameters. In the first of them the cut-off frequency was $166 \mathrm{~cm}^{-1}[6,7]$ and in the second $600 \mathrm{~cm}^{-1}[8,9]$. The first set accounts for strong system-bath coupling and low frequency modes of the bath. The second set accounts for a wider range of frequencies while the system-bath coupling is assumed to be small. We will present results with both sets.

Another parameter is the energy difference between the bridge and acceptor states, $\Delta \mathcal{G}_{21}=$ $H_{22}-H_{11}$. For wild-type bacteria $\Delta \mathcal{G}_{21}$ is thought to be in the range of $-480 \pm 180 \mathrm{~cm}^{-1}$ [41]. These values are inferred from analysis of magnetic data [42] for $\mathrm{P}^{+} \mathrm{BH}^{-}$and time-resolved data for the RCs obtained by site-directed mutagenesis [43]. In the calculations below we use the values of $\Delta \mathcal{G}_{21}$ in this range.

First we present the results obtained using recent electronic structure calculations [12], which estimated that $H_{12}=17 \mathrm{~cm}^{-1}$ and $H_{23}=122 \mathrm{~cm}^{-1}$ with the spectral density cut-off of $600 \mathrm{~cm}^{-1}$. To obtain an ET rate of about 3 ps we varied $\mathcal{G}_{21}$ and found that $\Delta \mathcal{G}_{21}=-450 \mathrm{~cm}^{-1}$ gives this rate. The data are graphed in Figure 1. The results are obtained using

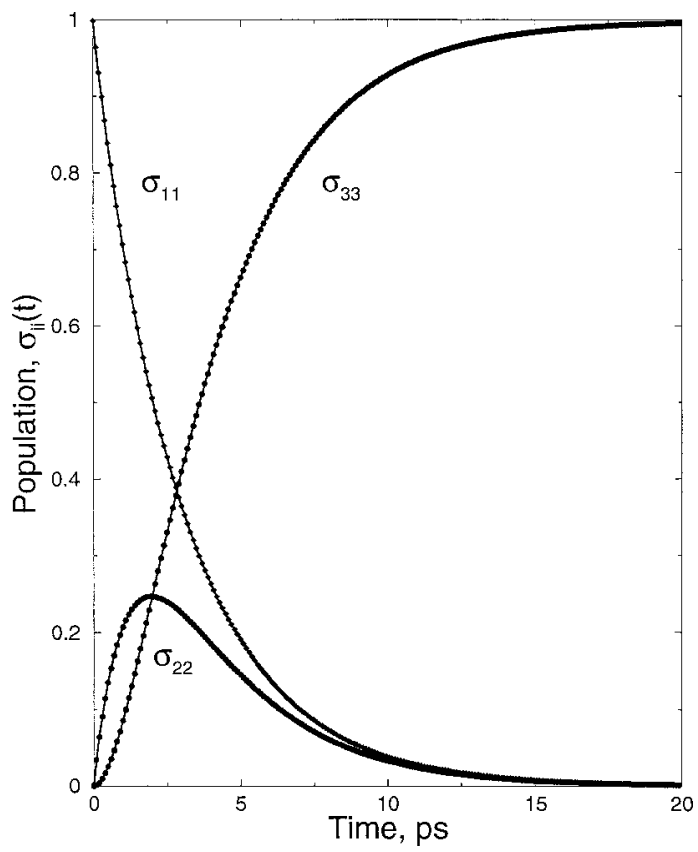

FIGURE 1. Population analysis of the ET reaction in RCs using couplings from electronic structure calculations [12], $H_{12}=17 \mathrm{~cm}^{-1}$ and $H_{23}=122 \mathrm{~cm}^{-1}$. $\Delta \mathcal{G}_{21}=-450 \mathrm{~cm}^{-1}$.

Davies' device, Eq. (25). As it is seen from the figure the simulations predict correct overall behavior of the reaction dynamics; i.e., the donor state decay rate is $\approx 3$ ps for $\Delta \mathcal{G}_{21}$ in the above indicated interval. Asymptotically the kinetics picture obtained using quantum mechanical correlation functions is also consistent with the classical results and satisfies the detailed balance condition. However, the maximum of the bridge population reaches $\approx 25 \%$ of the total population. This is larger than what has been predicted in experiments (maximum less than 20\%).

Figure 2 illustrates results obtained using coupling terms of $H_{12}=22 \mathrm{~cm}^{-1}$ and $H_{23}=135 \mathrm{~cm}^{-1}$ from recent dynamics simulations [8] in which the optimal value of $\Delta \mathcal{G}_{21}$ was found to be $-400 \mathrm{~cm}^{-1}$. In this case too, the overall dynamics behavior is consistent with the experimental results. However, differences appear at short-time dynamics. Thus, the population of the intermediate state, $P^{+} B^{-} H$, reaches a maximum at $\approx 37 \%$ by 2 ps and has a long-lasting tail. This dynamics behavior if present in the experiments should have been observable. However, as discussed above the signal from the $B^{-}$, when observed, is very weak. Therefore, according to our simulations the values of electronic coupling elements are slightly overestimated if $\Delta \mathcal{G}_{21}$ is set equal to $-400 \mathrm{~cm}^{-1}$. 


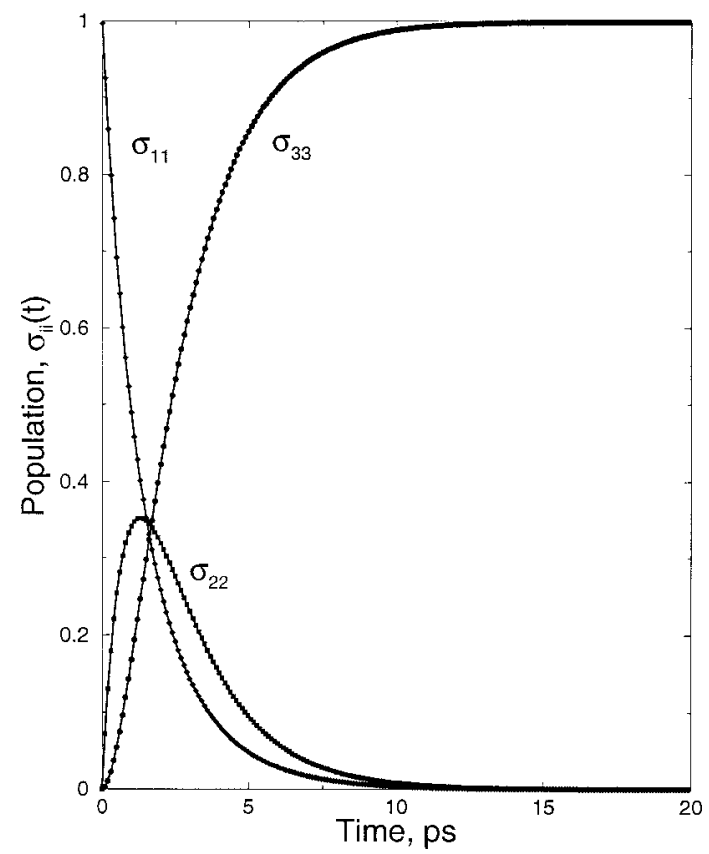

FIGURE 2. Time evolution of the diagonal elements of the density matrix from Davies' theory with

$H_{12}=22 \mathrm{~cm}^{-1}, H_{23}=135 \mathrm{~cm}^{-1}$, and

$\Delta \mathcal{G}_{21}=-400 \mathrm{~cm}^{-1}[9]$.

To predict dynamics behavior consistent with experimental observations, i.e., population of the bridge less than $20 \%$ of the total population, Davies' device suggests smaller intrasystem coupling terms. This is demonstrated in Figure 3 where the couplings are 125 and $20 \mathrm{~cm}^{-1}$ for $H_{12}$ and $H_{23}$, respectively. $\Delta \mathcal{G}_{21}$ was found to be $-550 \mathrm{~cm}^{-1}$. The $\mathrm{H}_{12} / \mathrm{H}_{23}$ ratio predicted in this study is in accord with the theoretical prediction of $6 \pm 2$ [44].

With an ohmic cut-off frequency of $600 \mathrm{~cm}^{-1}$ and a free energy of $\Delta \mathcal{G}_{31}=-2000 \mathrm{~cm}^{-1}$ for activationless electron transfer we can make the following conclusions based on results from Davies' device. $\mathrm{H}_{12}$ most strongly affects the decay rate of the donor state, $\sigma_{11}$, and to a large extent the maximum of the bridge population, $\sigma_{22}$, and the overall reaction rate as well. $\mathrm{H}_{23}$ mainly affects the population growth rate of the acceptor state, $\sigma_{33}$, the decay of the bridge state, and to a lesser degree the maximum population on the bridge state. A change of the acceptor-bridge gap $\left(\Delta \mathcal{G}_{21}\right)$ mainly affects the bridge population: an increase of $\Delta \mathcal{G}_{21}$ decreases the maximum bridge population.

We show only one figure obtained by using Redfield theory, Eq. (18). Figure 4 is typical of results from this method. It should be compared with the Figure 3 since all electronic structure and spectral

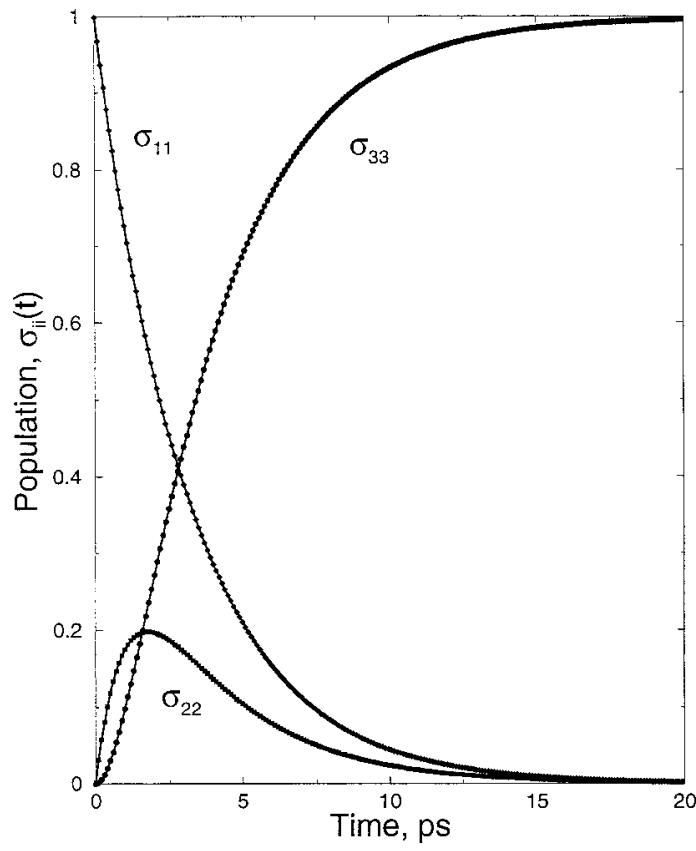

FIGURE 3. Density matrix time evolution with optimum parameters of Davies' device: $H_{12}=20 \mathrm{~cm}^{-1}$, $H_{23}=125 \mathrm{~cm}^{-1}$, and $\Delta \mathcal{G}_{21}=-550 \mathrm{~cm}^{-1}$.

density parameters are the same in both calculations. After a short propagation time the population numbers become negative. Furthermore, when quantum mechanical correlation functions of bath modes are used, for asymptotic times the dynamics does not agree with the classical asymptotes. The breakdown of the density matrix positivity in the original Redfield theory has been discussed before in the literature $[28,45]$.

Egger and Mak calculated reaction rates with a cut-off frequency of $166 \mathrm{~cm}^{-1}(\eta=6.036)$ and bridge energy, $\Delta \mathcal{G}_{21}$, varying between -666 and $+666 \mathrm{~cm}^{-1}$. They fixed the $H_{23} / H_{12}$ ratio at 4 . The authors also varied the reorganization energy to produce high values of the reaction rate. We are not able to reproduce fast ET with Davies' device for reasonable values of electronic structure parameters if the cut-off frequency is fixed at $166 \mathrm{~cm}^{-1}$. A characteristic result is demonstrated in Figure 5 where $H_{12}=38 \mathrm{~cm}^{-1}$ and $\Delta \mathcal{G}_{21}=-600 \mathrm{~cm}^{-1}$. We point out here that the apparant inability of Davies' device to produce a fast ET rate is due, in this case, to the low value of the cut-off frequency rather than to the large system-bath coupling (i.e., the breakdown of the second-order approximation). This is shown in Figure 6, in which we keep the Kondo factor, $\eta$, and the electronic couplings unchanged while $\omega_{\mathrm{c}}=300 \mathrm{~cm}^{-1}\left(\lambda_{\mathrm{r}}=3600 \mathrm{~cm}^{-1}\right)$. The difficulty is 


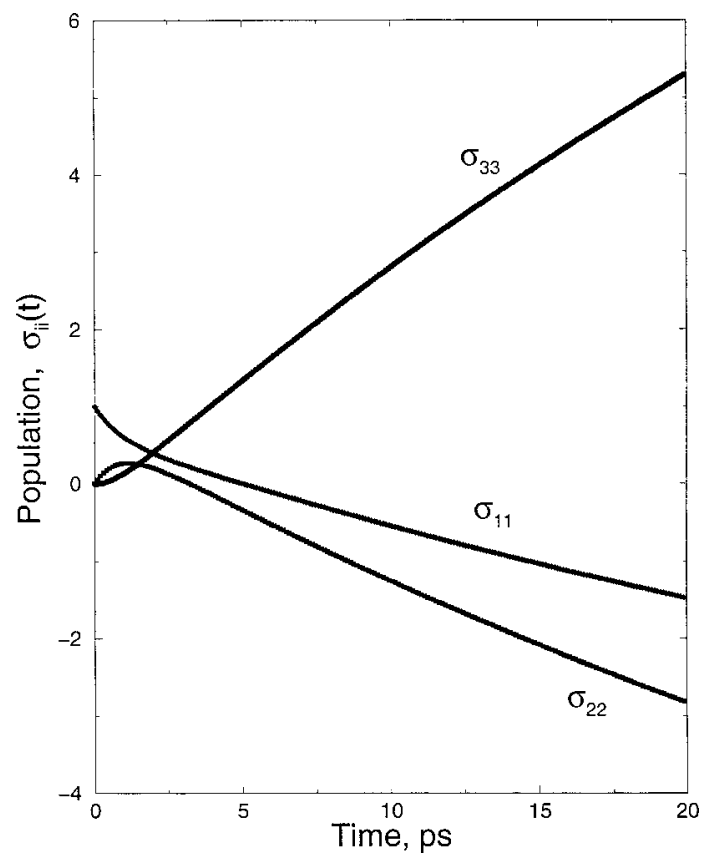

FIGURE 4. Results from the Redfield theory, with electronic structure and system-bath coupling elements the same as in Figure 3.

that at a low cut-off frequency the bath relaxation times are large and the Markovian approximation becomes invalid.

The electron transfer rate in primary charge separation of bacterial photosynthesis slightly increases by lowering the temperature [43]. For different bacteria the reaction rate is increased $2-5$ times in going from room temperature to the cryogenic temperatures. Most of the increase occurs up to $150 \mathrm{~K}$. Reorganization energies, free energies, and electronic and system-bath couplings are all affected by temperature. Experimental studies with mutated RCs had suggested that variations in free energies do not appear to increase the reaction rate sufficiently [43]. The observed acceleration of the ET was attributed mainly to the increase in the electronic coupling elements and/or a decrease in the reorganization energy. It has been suggested [46] that the main contributor to the rate increase is the contraction of the reaction centers which may reduce the centerto-center distances by up to $1 \AA$ and thus increase the electronic coupling terms by 2 -fold. A rigorous dynamics simulation should take account of these effects. So for our model of activationless ET with fixed electronic structure and system-bath coupling elements the reaction dynamics should not exhibit strong temperature dependence. This is what we find in Figure 7 where we depict the transient state

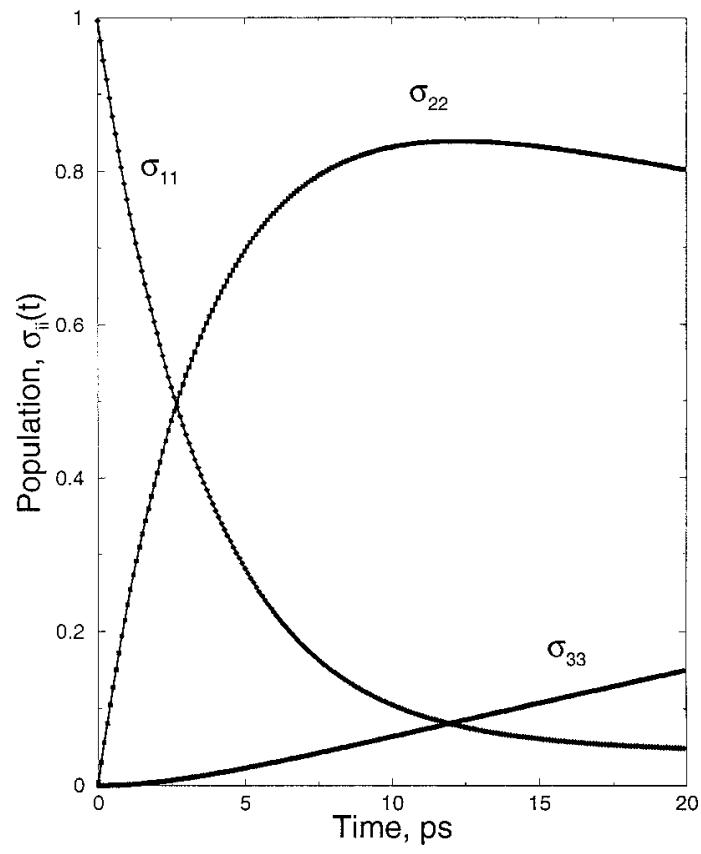

FIGURE 5. Transient populations in ET reaction with low cut-off frequency, $\omega_{\mathrm{c}}=166 \mathrm{~cm}^{-1}$, and $H_{12}=38 \mathrm{~cm}^{-1}, H_{23} / H_{12}=4$, and $\Delta \mathcal{G}_{23}=-600 \mathrm{~cm}^{-1}$.

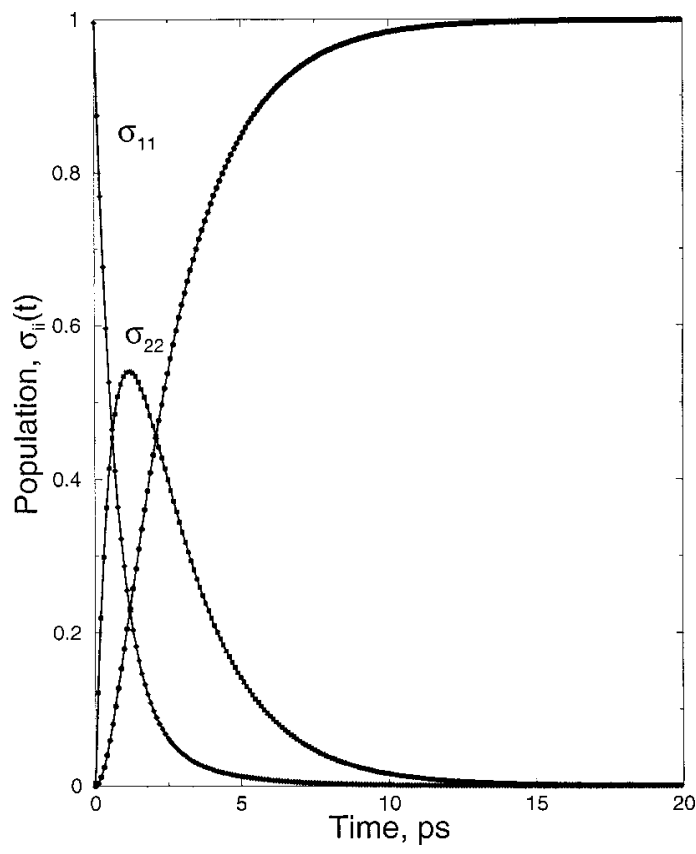

FIGURE 6. Results with the cut-off frequency of $300 \mathrm{~cm}^{-1}$. All other parameters are the same as in Figure 5. 


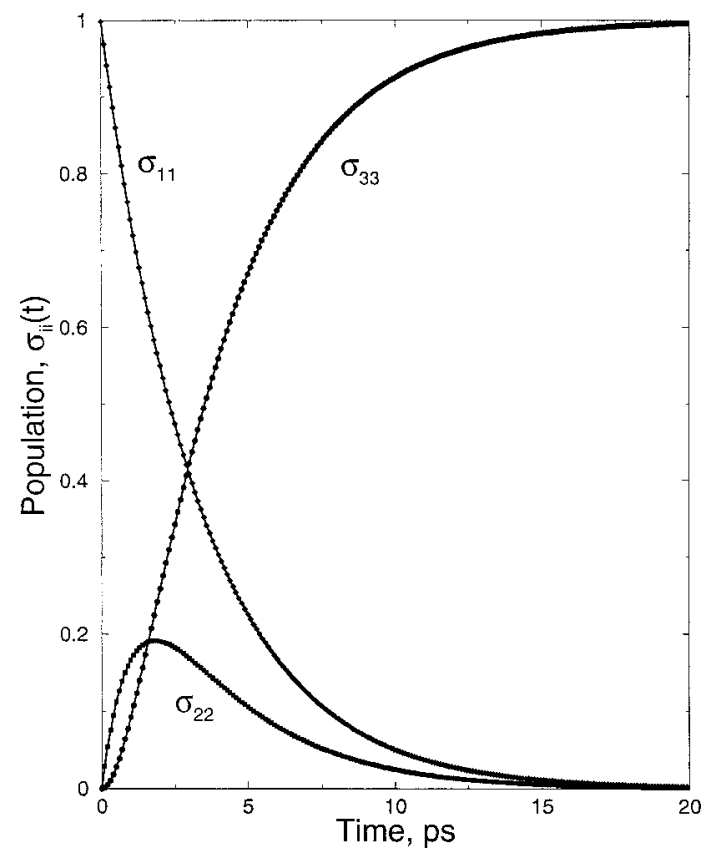

FIGURE 7. Low temperature, $T=150 \mathrm{~K}$, behavior of the ET dynamics as obtained from Davies' device. All parameters are the same as in Figure 3.

population computed with the same parameters as in Figure 3 but at $150 \mathrm{~K}$. The absence of strong temperature dependence of the reaction rate for the parameters employed is in agreement with the previous dynamical simulations $[8,9]$.

The power of the secular approximation is its gentle scaling with problem size. Once the system is cast in terms of adiabatic system states, the method scales formally as $N^{2}$ where $N$ is the number of system states (due to the coupling of the $i-k$ elements). In practice, if localized states are used, the scaling can be made even gentler, closer to linear in $N$. Thus, the secular approach can in principle be applied to very large systems. The results presented here verify the utility of the approach and its potential for larger systems. The only difficulty in the secular approach is with low-frequency bath motion, which can be handled by dividing the system into a low-frequency part to which the system responds adiabatically and a high-frequency part which is handled by the secular approximation. This and other partition strategies will be studied in future publications.

\section{Conclusions}

We have implemented the secular approximation, i.e., Davies' approach to Markovian dynamics to study primary charge separation processes in bacterial reaction centers. For a comparison, results obtained from the traditional Redfield relaxation theory are also presented. While Davies' formulation preserves the positive-definite nature of the density matrix, the Redfield theory breaks it down. Therefore Davies' device is suggested to be a useful tool to study charge separation process happening in Markovian limit. The results agree well with the previous theoretical calculations and experiments where data are available. Long-time dynamics and the time dependence of activationless KT in simulations confirm the results of recent studies. Differences emerge in short-time dynamics, where we predict a suitable set of electronic coupling parameters to promote efficient ET.

\section{ACKNOWLEDGMENTS}

This work was supported by the NSF, the PRF, the Sloan Foundation and the UCLA Academic Senate.

\section{References}

1. Deisenhofer, J.; Epp, O.; Miki, K.; Huber, R.; Michel, H. J Mol Biol 1984, 180, 385.

2. Chan, C.-K.; DiMagno, T. J.; Chen, L. Q.-X.; Norris, J. R.; Flemming, G. R. Proc Natl Acad Sci USA 1991, 88, 11202.

3. Kirmaier, C.; Holten, D. Proc Natl Acad Sci USA 1990, 87, 3552.

4. Thompson, M. A.; Zerner, M. C. J Am Chem Soc 1991, 113, 8210.

5. Marchi, M.; Gehlen, J. N.; Chanler, D. J Am Chem Soc 1993, 115,4178

6. Egger, R.; Mak, C. H. J Phys Chem 1994, 98, 9903.

7. Mak, C. H.; Egger, R. Chem Phys Lett 1995, 238, 149.

8. Makri, N.; Sim, E.; Makarov, D. E.; Topaler, M. Proc Natl Acad Sci USA 1996, 93, 3926.

9. Sim, E.; Makri, N. J Phys Chem B 1997, 101, 5446.

10. Makarov, D. E.; Makri, N. Phys Rev A 1993, 48, 3626.

11. Makri, N.; Makarov, D. E. J Chem Phys 1995, 102, 4600.

12. Zhang, L. Y.; Friesner, R. A. Proc Natl Acad Sci USA 1998, 95, 13603 .

13. Golosov, A. G.; Friesner, R. A.; Pechukas, P. J Chem Phys 1999, 110, 138.

14. Thompson, K.; Makri, N. J Chem Phys 1999, 110, 1343.

15. Redfield, A. IBM J Res Dev 1957, 1, 19.

16. Redfield, A. G. Adv Mag Reson 1965, 1, 1.

17. Pollard, W. T.; Felts, A. K.; Friesner, R. A. J Phys Chem 1995, 99, 2929.

18. Okada, A.; Chernyak, V.; Mukamel, S. J Phys Chem A 1998, 102,1241

19. Kohen, D.; Tannor, D. J. J Chem Phys 1997, 107, 5141. 
20. Walsh, A. M.; Coalson, R. D. Chem Phys Lett 1992, 195, 293.

21. Felts, A. K.; Pollard, W. T.; Friesner, R. A. J Phys Chem 1995, 2929.

22. Jean, J. M.; Friesner, R. A.; Flemming, G. R. J Chem Phys 1992, 96, 5827.

23. Davies, E. Commun Math Phys 1974, 39, 91.

24. Davies, E. B. Quantum Theory of Open Systems; Academic Press: New York, 1976.

25. Leggett, A. J.; Chakravarty, S.; Dorsey, A. T.; Fisher, M. P. A.; Garg, A.; Zwerger, W. Rev Mod Phys 1987, 59, 1.

26. Zubarev, D. N. Nonequilibrium Statistical Thermodynamics; Consultants Bureau, 1974.

27. Ogrodnik, A. Biochim Biophys Acta 1990, 1020.

28. Pollard, W. T.; Felts, A. K.; Friesner, R. A. Adv Chem Phys 1996, 93, 77.

29. van Kampen, N. G. Stochastic Processes in Physics and Chemistry; North-Holland: Amsterdam, 1992.

30. Redfield, A. Phys Rev 1955, 98, 1787.

31. Blum, K. Density Matrix Theory and Applications; Plenum Press: New York, 1981.

32. Wertheimer, R.; Silbey, R. Chem Phys Lett 1980, 75, 243.

33. Dumcke, R.; Spohn, H. Z Phys B 1979, 34, 419.

34. Davies, E. B. Math Ann 1976, 219, 147.
35. Lindblad, G. Commun Math Phys 1975, 40, 147.

36. Alicki, R.; Lendi, K. Quantum Dynamical Semigroups and Applications; Springer-Verlag: New York, 1987.

37. Tal-Ezer, H.; Kosloff, R. J Chem Phys 1984, 81, 3967.

38. Neuhauser, D.; Baer, M.; Judson, R. S.; Kouri, D. J. Comput Phys Commun 1991, 63, 460.

39. Huisinga, W.; Pesce, L.; Kozloff, R.; Saalfrank, P. J Chem Phys 1999, 110, 5538.

40. Guo, H.; Chen, R. J Chem Phys 1999, 110, 6626.

41. Bixon, M.; Jortner, J.; Michel-Beyerle, M. E. Chem Phys Lett 1995, 197, 389.

42. Volk, M.; Ogrodnik, A.; Michel-Beyerle, M. E. Spacings between Bridge and Donor States. In Anoxygenic Photosynthetic Bacteria; Blakenship, R. E.; Medigan, M. T.; Bauer, C. E., Eds.; Kluwer Academic Publishers: Dordrecht, The Netherlands, 1995.

43. Huber, H.; Meyer, M.; Scheer, H.; Zinth, W.; Wachtveitl, J. Photosyn Res 1998, 55, 153.

44. Plato, M.; Mobius, K.; Michel-Beyerle, M. E.; Bixon, M.; Jortner, J. J Am Chem Soc 1988, 110, 7279.

45. Suarez, A.; Silbey, R.; Oppenheim, I. J Chem Phys 1992, 97, 5101.

46. Zinth, W.; Huppmann, P.; Arlt, T.; Wachtveitl, J. Philos Trans R Soc London Ser A 1998, 356, 465. 